\title{
Platelet/lymphocyte ratio and mean platelet volume in patients with granulomatosis with polyangiitis
}

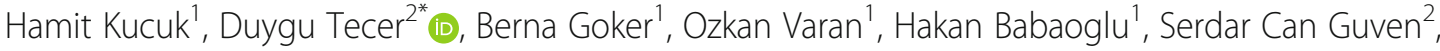 \\ Mehmet Akif Ozturk', Seminur Haznedaroglu' ${ }^{1}$ and Abdurrahman Tufan ${ }^{1}$
}

\begin{abstract}
Background: Granulomatosis with polyangiitis (GPA) is a granulomatous necrotizing vasculitis with high morbidity and mortality. Anti-neutrophil cytoplasmic antibody is a valuable diagnostic marker, however its titer lacks predictive value for the severity of organ involvement. Platelet to lymphocyte ratio (PLR) and mean platelet volume (MPV) has been regarded as a potential marker in assessing systemic inflammation. We aimed to explore the value of PLR and MPV in the assessment of disease activity and manifestations of disease in GPA.

Methods: 56 newly diagnosed GPA patients and 53 age-sex matched healthy controls were included in this retrospective and cross-sectional study with comparative group. Complete blood count was performed with Backman Coulter automatic analyzer, erythrocyte sedimentation rate (ESR) with Westergen method and C-reactive protein (CRP) levels with nephelometry. The PLR was calculated as the ratio of platelet and lymphocyte counts.

Result: Compared to control group, ESR, CRP and PLR were significantly higher and MPV significantly lower in GPA patients. In patients group, PLR was positively correlated with ESR and CRP $(r=0.39, p=0.005$ and $r=0.51, p<0.001$, respectively). MPV was negatively correlated with ESR and CRP $(r=-0.31, p=0.028$ and $r=-0.34 p=0.014$, respectively). Patients with renal involvement had significantly higher PLR than patients without renal involvement (median:265.98, IQR:208.79 vs median:180.34 IQR:129.37, $p=0.02$ ). PLR was negatively correlated with glomerular filtration rate $(r=-0.27, p=0.009)$. A cut-off level of 204 for PLR had $65.6 \%$ sensitivity and 62.5 specificity to predict renal involvement.
\end{abstract}

Conclusion: PLR exhibit favorable diagnostic performance in predicting renal involvement in patients with GPA.

Keywords: Granulomatosis with polyangiitis, Platelet to lymphocyte ratio, Mean platelet volume, Biomarker, Activity

\section{Background}

Granulomatosis with polyangiitis (GPA), formerly known as Wegener's granulomatosis (WG) is an autoimmune vasculitis characterized by granulomatous inflammation with necrotizing vasculitis affecting small to medium sized vessels [1]. The main autoantibody associated with the disease is the cytoplasmic antineutrophil cytoplasmic antibodies (c-ANCA), usually directed against the enzyme proteinase-3 (PR-3) [2]. Due to involvement of vital organs, GPA has significant morbidity

\footnotetext{
* Correspondence: duygu-tecer@hotmail.com

${ }^{2}$ Faculty of Medicine, Department of Physical Medicine \& Rehabilitation,

Division of Rheumatology, Gazi University, Ankara, Turkey

Full list of author information is available at the end of the article
}

and mortality. Renal involvement observed as rapidly progressive glomerulonephritis may lead to end-stage renal failure. Other potentially fatal common manifestations are alveolar and gastrointestinal hemorrhage and myocarditis. With the advanced treatment regimens, the disease has become more of a chronic relapsing-remitting pattern. Relapses occur in $50 \%$ or more of patients during the long-term follow-up [3]. One of the major challenges in the management of GPA is lack of reliable markers for activity and predicting relapse to guide therapy. Moreover, association between initial presentation features and subsequent relapses are controversial [4]. Because of ANCA titres and conventional inflammation markers such as C-reactive protein (CRP) and erythrocyte

(C) The Author(s). 2019 Open Access This article is distributed under the terms of the Creative Commons Attribution 4.0 International License (http://creativecommons.org/licenses/by/4.0/), which permits unrestricted use, distribution, and 
sedimentation rate (ESR) have limited value, new biomarkers are needed for the assessment of disease activity, and to predict relapse [5-7].

Platelet to lymphocyte ratio (PLR) is absolute count of platelets divided by the absolute count of lymphocytes derived from routine complete blood count (CBC). PLR was emerged as a marker of inflammation and used in combination with other inflammatory markers to determine severity of inflammation in many diseases. In recent years, utility of PLR was evaluated in numerous studies including cancers, cardiovascular diseases, rheumatic disease [8-11]. As novel markers of inflammation, we aim to investigate utility of PLR and mean platelet volume (MPV) in the assessment of disease activity and manifestations of disease in patients with GPA.

\section{Methods}

This study was planned as the retrospective and crosssectional study with comparative group. 56 patients with GPA who were diagnosed between 2012 and 2017 were included. All patients met the Chapel Hill Consensus Conference Nomenclature/Criteria for Vasculitis and/or the American College of Rheumatology (ACR) criteria for GPA [12, 13]. 53 age and sex-matched healthy subjects were served as control group. Subjects in either group with one of the following concomitant diseases/situations were excluded: 1) acute or chronic infections; 2) concomitant inflammatory diseases 3) metabolic diseases including diabetes mellitus, thyroid dysfunction, liver disease and 4) any kind of malignancy.

Demographic, clinical and laboratory data were retrieved from medical records. Disease activity was assessed with Birmingham Vasculitis Activity Score for WG vasculitis (BVAS/WG) [14]. Blood collection and calculation of BVAS/ WG were held at the same time. All blood samples were collected from newly diagnosed patients. Complete blood count (CBC) was performed with Backman Coulter automatic analyzer within $2 \mathrm{~h}$ of blood collection. ESR and CRP levels were determined with Westergen method and nephelometry respectively. The PLR was calculated as the ratio of platelet and lymphocyte counts of the same CBC. Renal involvement was diagnosed if patient had at least one of the following findings: a. active, biopsy proven, pauci-immune glomerulonephritis, b. active urinary sediment, c. rise in serum creatinine $>30 \%$ or $>25 \%$ decline in creatinine clearance which was attributed to active AAV in the kidney. The study protocol was approved by the Local Research Ethics Committee. A written informed consent form was signed by the all participants. Study was conducted in accordance with the ethical principles as described by the declaration of Helsinki.

\section{Statistical analysis}

Statistical Package for Social Science (SPSS) version of 16.0 was used for the analyzes (SPSS Inc., Chicago, IL). The variables were analyzed using visual (histograms, probability plots) and analytical methods (KolmogorovSmirnov) for the distribution of normality. All demographic and quantitative data were presented as means \pm SD or percentages (\%). Comparison of categorical data was performed by chi-square tests. Mann-Whitney Utest was used to compare independent samples which did not have a normal distribution. A $p$-value $<0.05$ were considered statistically significant. Spearman test was used for the assessment of correlations between variables. Sensitivity, specificity and cut off values are determined by using ROC curve and diagram. Comparison of ROC curves were used for comparing predictive performances of RDW, ESR and CRP variables to detect renal involvement.

\section{Results}

Fifty-six patients with GPA and 53 healthy controls were included. Clinical characteristics and laboratory findings of the study groups are shown in Table 1 . The patients were predominantly male $(58.9 \%)$ with a mean age of $48.14 \pm 14.09$ years. C-ANCA was positive in 51 (91.07\%) of patients and p-ANCA was positive in $8(16.07 \%)$ of patients. Mean BVAS/WG was $13.54 \pm 4.94$ at diagnosis. Clinical manifestations at diagnosis was as follow, general manifestation 51 (91.07\%), ear nose throat involvement 33 (58.93\%), pulmonary involvement 37 (66.1\%), renal involvement $32(57.1 \%)$, cutaneous involvement $25(44.64 \%)$, ocular 17 (30.36\%), gastrointestinal tract 5 (8.93\%).

ESR, CRP and PLR were significantly higher in patients with GPA than controls. MPV was significantly lower in patients with GPA compared to healthy controls. In patients group, PLR positively correlated with ESR and CRP $(r=0.39, p=0.005$ and $r=0.51, p<0.001$, respectively). In contrast, MPV negatively correlated with ESR and CRP $(r=-0.31, p=0.03$ and $r=-0.34, p=0.014$, respectively). There were no significant correlations between PLR, MPV and BVAS/WG.

Patients with renal involvement had remarkably higher PLR than patients without renal involvement (median: 265.98, IQR: 208.79 vs median: 180.34 IQR:129.37, $p=$ 0.02). Moreover, PLR negatively correlated with glomerular filtration rate $(r=-0.27, p=0.009)$. Patients with renal involvement tended to have lower MPV, but this difference did not reach statistical significance (median: 7.60, IQR:1.17 vs median 7.75, IQR:1.46, $p=0.786$ ). Receiver operating characteristic curve of PLR, ESR and CRP for differentiating renal involvement is presented in Fig. 1. Area Under Curves (AUCs) for PLR, CRP and ESR were 0.703 (95\% confidence interval [CI], 0.558$0.849, p=0.016$ ), 0.577 (95\% CI: 0.416-0.738, $p=0.362$ ), 0.508 (95\% CI: $0.337-0.678, p=0.929$ ), respectively. A cut-off level of 204 for PLR had $65.6 \%$ sensitivity and 62.5 specificity (positive predictive value $70 \%$, negative 
Table 1 Clinical characteristics and laboratory findings of the study population

\begin{tabular}{llll}
\hline & GPA patients & Controls & $p$ \\
\hline Age mean \pm SD (years) & $48.14 \pm 14.09$ & $46.77 \pm 14.14$ & 0.614 \\
Males $(\mathrm{n})$ & $33(58.9 \%)$ & $26(49.1 \%)$ & 0.301 \\
WBC $\left(\times 10^{3} / \mathrm{mL}\right)$ & $11.600(3.240 ; 35.490 ; 6.907)$ & $7.040(4.100 ; 11.870 ; 2.413)$ & $<0.001$ \\
Neutrophils $\left(\times 10^{3} / \mathrm{mL}\right)$ & $8.230(1.300 ; 33.420 ; 6.830)$ & $4000(2.300 ; 11.870 ; 1.525)$ & $<0.001$ \\
Lymphocytes $\left(\times 10^{3} / \mathrm{mL}\right)$ & $1.665(0.300 ; 5.550 ; 0.951)$ & $2.320(1.270 ; 4.090 ; 0.690)$ & $<0.001$ \\
Platelets $\left(\times 10^{3} / \mathrm{mL}\right)$ & $308.000(82.600 ; 1126.000 ; 191.500)$ & $224.600(136.000 ; 373.900 ; 73.600)$ & $<0.001$ \\
ESR $(\mathrm{mm} / \mathrm{H})$ & $47.5(3 ; 131 ; 60)$ & $8(1 ; 34 ; 7.50)$ & $3.1(1.16 ; 7.40 ; 2.07)$ \\
CRP $(\mathrm{mg} / \mathrm{L})$ & $34(1.27 ; 300 ; 99.14)$ & $8.62(6.88 ; 13.10 ; 1.41)$ & $<0.001$ \\
MPV & $7.54(6.08 ; 10.38 ; 1.29)$ & $101.43(58.37 ; 210.63 ; 35.44)$ & $<0.001$ \\
PLR & $212.65(29.86 ; 1638.33 ; 159.62)$ & $0.79(0.53 ; 1.12 ; 0.24)$ & $<0.001$ \\
Creatinine $(\mathrm{mg} / \mathrm{dL})$ & $0.912(0.45 ; 19.90 ; 1)$ & $99.70(65.30 ; 130.70 ; 21.30)$ & $<0.001$ \\
GFR & $83.50(2.70 ; 141.0 ; 75.25)$ & 0.002 & $<$ \\
\hline
\end{tabular}

Values are presented as median (min; max; interquartile range). GPA Granulomatosis with polyangiitis, WBC white blood cell, ESR erythrocyte sedimentation rate, $C R P$ C-reactive protein, MPV mean platelet volume, PLR platelet/lymphocyte ratio, GFR glomerular filtration ratio

predictive value 57.7\%) for renal involvement (Fig. 2). Patients with alveolar hemorrhage tended to have higher PLR and lower MPV but this difference did not reach statistical significance $(277.34 \pm 181.95$ vs $240.61 \pm$ $252.43 p=0.382$ for PLR, $7.69 \pm 0.66$ vs $7.83 \pm 1.08, p=$ 0.809 for MPV, respectively).

\section{Discussion}

Significant progress has been made in the understanding of pathogenesis and treatment of GPA, but there is still unmet need for biomarkers for predicting specific organ involvement, disease activity, relapse and long term prognosis. Identification of such markers may guide the

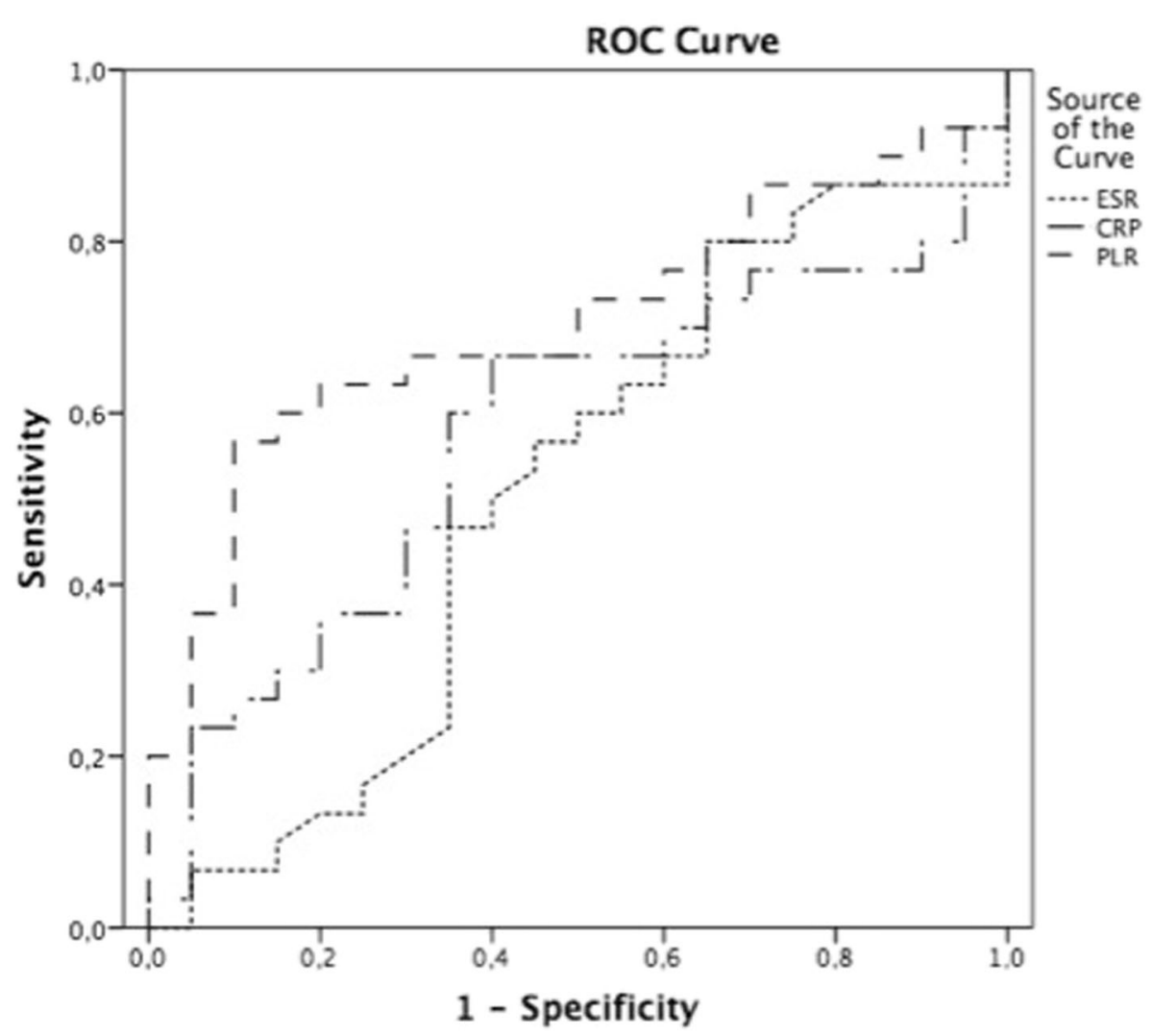

Fig. 1 Receiver operating characteristic curve of PLR, ESR and CRP for differentiating renal involvement 


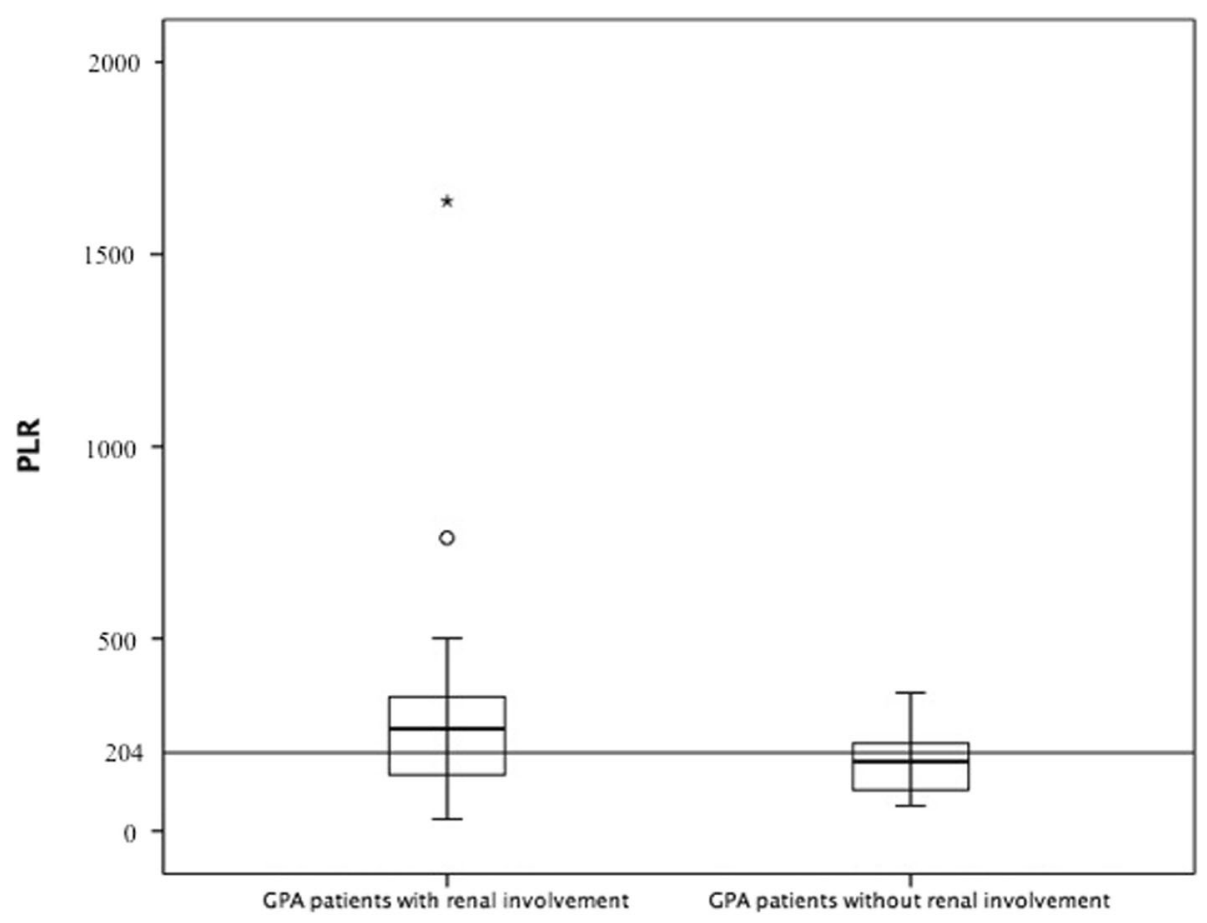

Fig. 2 Characteristics of PLR

therapy and help to determine those patients at high risk of relapse. Available markers, including ANCA titers, and commonly used inflammatory markers, ESR and CRP, are not adequate. Although these markers substantially elevated in active stages of disease their values are not correlated with disease activity and lack of prognostic information and prediction of relapses.

Systemic inflammation is associated with alterations in circulating peripheral blood cells quantity and composition. Normochromic anemia, thrombocytosis, neutrophilia and lymphocytopenia usually accompanies many inflammatory conditions [15]. In acute inflammation number and volume of platelets increase. Therefore, these features of circulating blood cell components can be used for the assessment of inflammatory activity [16, 17]. One of these, PLR has emerged as a marker of activity and as a prognostic marker in many diseases.

In our study, we found that PLR is significantly elevated in patients with GPA and correlated with other commonly used acute phase reactants. There is no correlation between PLR and BVAS/WG indicating that PLR might not reflect overall activity of disease. However, those patients with renal involvement had remarkably higher levels and PLR was significantly correlated with GFR. Therefore, we suggest that PLR might be a marker for renal activity of GPA.

In recent years, utility of PLR was evaluated in numerous studies. It has been reported to be elevated in patients with chronic renal failure (CRF) and is associated with increased mortality among the end stage CRF patients $[18,19]$. Another striking evidence is correlation between PLR and disease activity of dermatomyositis which is widely accepted as a vasculitic process [20]. In addition, previous studies show that PLR is positively correlated with inflammatory indices such as CRP and ESR, and PLR is also associated with disease activity in psoriasis, RA and systemic lupus erythematosus [21-24]. Systemic lupus erythematosus (SLE) patients with nephritis had higher PLR levels than those without nephritis [24].

MPV was significantly lower in patients with GPA compared to healty control and negatively correlated witih ESR and CRP. There was no correlation between BVAS/WG, GFR and MPV. MPV values have previously been studied in various inflammatory conditions, such as familial mediterranean fever, rheumatoid arthritis, ankylosing spondylitis, inflammatory bowel disease, juvenile SLE, psoriasis, systemic sclerosis and acute rheumatic fever [16, 25-31]. But the results are contradictory. It would seem that the size of circulating platelets is dependent on the intensity of systemic inflammation. Low-grade inflammatory conditions are associated with high levels of MPV and high-grade inflammatory diseases are associated with low levels of MPV [32, 33].

Herein, we presented 56 patients with GPA and tried to analyze the relation between PLR, MPV and disease activity. These costs may change from country to country, but the cost-effectiveness of these new parameters is valid worldwide. We have some limitations in our study. 
First, our study is cross sectional and long term prognosis of patients are largely unknown. Second, number of patients is relatively small. Hence, our results must be confirmed in large scale longitudinal prospective studies.

\section{Conclusions}

Patients with GPA had significantly higher PLR and lower MPV compared to healthy controls. We have demonstrated a significant correlation between ESR, CRP, MPV and PLR. GPA patients with renal involvement had higher PLR levels than those without renal involvement and PLR was significantly correlated with GFR. Newer biomarkers detected in urine or blood could greatly assist with diagnosis, disease activity assessment, and prognosis of patients with GPA; however, at present there is a need for prospective and longitudinal studies followed by validation in different groups of GPA patients to confirm their clinical value [34].

\section{Abbreviation}

ACR: American College of Rheumatology; ANCA: Antineutrophil cytoplasmic antibodies; BVAS:MG: Birmingham Vasculitis Activity Score for WG vasculitis; CBC: Complete blood count; CRP: C-reactive protein; ESR: Erythrocyte sedimentation rate; GPA: Granulomatosis with polyangiitis; IQR: Inter-quartile range; MPV: Mean platelet volume; PLR: Platelet to lymphocyte ratio; PR3: Proteinase-3; SD: Standard deviation; SPSS: Statistical Package for Social Science; WG: Wegener's granulomatosis

\section{Acknowledgements}

None

\section{Authors' contributions}

All of the authors declare that they have all participated in the design, execution, and analysis of the paper, and that they have approved the final version.

\section{Funding}

This research did not receive any specific grant from funding agencies in the public, commercial, or not-for-profit sectors.

\section{Availability of data and materials}

The datasets used and/or analysed during the current study are available from the corresponding author on reasonable request.

\section{Ethics approval and consent to participate}

The study protocol was approved by the Committee on Human Research Ethics

\section{Consent for publication}

A well written informed consent and consent to publish was obtained from all the partipicants included in this study.

\section{Competing interests}

All co-authors are aware of your journal's conflict-of-interest policy; none of the co-authors has any direct or indirect conflicts of interest, financial or otherwise, relating to the subject of our report.

\section{Author details}

${ }^{1}$ Faculty of Medicine, Department of Internal Medicine, Division of Rheumatology, Gazi University, Ankara, Turkey. ${ }^{2}$ Faculty of Medicine, Department of Physical Medicine \& Rehabilitation, Division of Rheumatology, Gazi University, Ankara, Turkey.
Received: 13 October 2018 Accepted: 23 December 2019

Published online: 31 December 2019

\section{References}

1. Pagnoux C. Updates in ANCA-associated vasculitis. Eur J Rheumatol. 2016; 3(3):122-33.

2. van der Woude FJ, Rasmussen N, Lobatto S, Wiik A, Permin H, van Es LA, et al. Autoantibodies against neutrophils and monocytes: tool for diagnosis and marker of disease activity in Wegener's granulomatosis. Lancet. 1985; 1(8426):425-9.

3. Weiner $M$, Segelmark $M$. The clinical presentation and therapy of diseases related to anti-neutrophil cytoplasmic antibodies (ANCA). Autoimmun Rev. 2016:15(10):978-82

4. Pagnoux C, Hogan SL, Chin H, Jennette JC, Falk RJ, Guillevin L, et al. Predictors of treatment resistance and relapse in antineutrophil cytoplasmic antibody-associated small-vessel vasculitis: comparison of two independent cohorts. Arthritis Rheum. 2008;58(9):2908-18.

5. Verstockt B, Bossuyt X, Vanderschueren S, Blockmans D. There is no benefit in routinely monitoring ANCA titres in patients with granulomatosis with polyangiitis. Clin Exp Rheumatol. 2015;33(2 Suppl 89):S-72-6.

6. Tomasson G, Grayson PC, Mahr AD, Lavalley M, Merkel PA. Value of ANCA measurements during remission to predict a relapse of ANCA-associated vasculitis--a meta-analysis. Rheumatology (Oxford). 2012;51(1):100-9.

7. Thai $\mathrm{LH}$, Charles P, Resche-Rigon M, Desseaux K, Guillevin L. Are antiproteinase-3 ANCA a useful marker of granulomatosis with polyangiitis (Wegener's) relapses? Results of a retrospective study on 126 patients. Autoimmun Rev. 2014;13(3):313-8.

8. Zhang M, Huang XZ, Song YX, Gao P, Sun JX, Wang ZN. High platelet-tolymphocyte ratio predicts poor prognosis and Clinicopathological characteristics in patients with breast Cancer: a meta-analysis. Biomed Res Int 2017:2017:9503025.

9. Idil Soylu A, Arikan Cortcu S, Uzunkaya F, Atalay YO, Bekci T, Gungor L, et al. The correlation of the platelet-to-lymphocyte ratio with the severity of stenosis and stroke in patients with carotid arterial disease. Vascular. 2017;25(3):299-306.

10. Prodromidou A, Andreakos P, Kazakos C, Vlachos DE, Perrea D, Pergialiotis V. The diagnostic efficacy of platelet-to-lymphocyte ratio and neutrophil-toIymphocyte ratio in ovarian cancer. Inflamm Res. 2017;66(6):467-75.

11. Zhu Y, Si W, Sun Q, Qin B, Zhao W, Yang J. Platelet-lymphocyte ratio acts as an indicator of poor prognosis in patients with breast cancer. Oncotarget. 2017;8(1):1023-30

12. Jennette JC, Falk RJ, Andrassy K, Bacon PA, Churg J, Gross WL, et al. Nomenclature of systemic vasculitides. Proposal of an international consensus conference. Arthritis Rheum. 1994;37(2):187-92.

13. Leavitt RY, Fauci AS, Bloch DA, Michel BA, Hunder GG, Arend WP, et al. The American College of Rheumatology 1990 criteria for the classification of Wegener's granulomatosis. Arthritis Rheum. 1990;33(8):1101-7.

14. Stone JH, Hoffman GS, Merkel PA, Min Yl, Uhlfelder ML, Hellmann DB, et al. A disease-specific activity index for Wegener's granulomatosis: modification of the Birmingham Vasculitis activity score. International network for the study of the systemic Vasculitides (INSSYS). Arthritis Rheum. 2001;44(4):912-20.

15. Gabay C, Kushner I. Acute-phase proteins and other systemic responses to inflammation. N Engl J Med. 1999:340(6):448-54.

16. Kisacik B, Tufan A, Kalyoncu U, Karadag O, Akdogan A, Ozturk MA, et al. Mean platelet volume (MPV) as an inflammatory marker in ankylosing spondylitis and rheumatoid arthritis. Joint Bone Spine. 2008;75(3):291-4

17. Kucuk H, Goker B, Varan O, Dumludag B, Haznedaroglu S, Ozturk MA, et al. Predictive value of neutrophil/lymphocyte ratio in renal prognosis of patients with granulomatosis with polyangiitis. Ren Fail. 2017;39(1):273-6.

18. Ahbap E, Sakaci T, Kara E, Sahutoglu T, Koc Y, Basturk T, et al. Neutrophil-tolymphocyte ratio and platelet-tolymphocyte ratio in evaluation of inflammation in end-stage renal disease. Clin Nephrol. 2016;85(4):199-208.

19. Yaprak M, Turan MN, Dayanan R, Akin S, Degirmen E, Yildirim M, et al. Plateletto-lymphocyte ratio predicts mortality better than neutrophil-to-lymphocyte ratio in hemodialysis patients. Int Urol Nephrol. 2016:48(8):1343-8.

20. Yang $W$, Wang $X$, Zhang $W$, Ying $H, X u Y$, Zhang J, et al. Neutrophillymphocyte ratio and platelet-lymphocyte ratio are 2 new inflammatory markers associated with pulmonary involvement and disease activity in patients with dermatomyositis. Clin Chim Acta. 2017:465:11-6.

21. Kim DS, Shin D, Lee MS, Kim HJ, Kim DY, Kim SM, et al. Assessments of neutrophil to lymphocyte ratio and platelet to lymphocyte ratio in Korean patients with psoriasis vulgaris and psoriatic arthritis. J Dermatol. 2016;43(3):305-10. 
22. Asahina A, Kubo N, Umezawa Y, Honda H, Yanaba K, Nakagawa $\mathrm{H}$. Neutrophil-lymphocyte ratio, platelet-lymphocyte ratio and mean platelet volume in Japanese patients with psoriasis and psoriatic arthritis: response to therapy with biologics. J Dermatol. 2017;44(10):1112-21.

23. Fu H, Qin B, Hu Z, Ma N, Yang M, Wei T, et al. Neutrophil- and platelet-tolymphocyte ratios are correlated with disease activity in rheumatoid arthritis. Clin Lab. 2015;61(3-4):269-73.

24. Qin B, Ma N, Tang Q, Wei T, Yang M, Fu H, et al. Neutrophil to lymphocyte ratio (NLR) and platelet to lymphocyte ratio (PLR) were useful markers in assessment of inflammatory response and disease activity in SLE patients. Mod Rheumatol. 2016;26(3):372-6.

25. Yavuz S, Ece A. Mean platelet volume as an indicator of disease activity in juvenile SLE. Clin Rheumatol. 2014;33(5):637-41.

26. Ozdemir R, Karadeniz C, Doksoz O, Celegen M, Yozgat Y, Guven B, et al. Are mean platelet volume and platelet distribution width useful parameters in children with acute rheumatic carditis? Pediatr Cardiol. 2014;35(1):53-6.

27. Karabudak O, Ulusoy RE, Erikci AA, Solmazgul E, Dogan B, Harmanyeri Y. Inflammation and hypercoagulable state in adult psoriatic men. Acta Derm Venereol. 2008;88(4):337-40.

28. Abanonu GB, Daskin A, Akdogan MF, Uyar S, Demirtunc R. Mean platelet volume and beta-thromboglobulin levels in familial Mediterranean fever: effect of colchicine use? Eur J Intern Med. 2012;23(7):661-4.

29. Ozturk ZA, Dag MS, Kuyumcu ME, Cam H, Yesil Y, Yilmaz N, et al. Could platelet indices be new biomarkers for inflammatory bowel diseases? Eur Rev Med Pharmacol Sci. 2013;17(3):334-41.

30. Soydinc S, Turkbeyler IH, Pehlivan Y, Soylu G, Goktepe MF, Bilici M, et al. Mean platelet volume seems to be a valuable marker in patients with systemic sclerosis. Inflammation. 2014;37(1):100-6.

31. Tecer D, Sezgin M, Kanik A, Incel NA, Cimen OB, Bicer A, et al. Can mean platelet volume and red blood cell distribution width show disease activity in rheumatoid arthritis? Biomark Med. 2016;10(9):967-74.

32. Balta I, Balta S, Koryurek OM, Demirkol S, Celik T, Akbay G, et al. Mean platelet volume is associated with aortic arterial stiffness in patients with Behcet's disease without significant cardiovascular involvement. J Eur Acad Dermatol Venereol. 2014;28(10):1388-93.

33. Gasparyan AY, Ayvazyan L, Mikhailidis DP, Kitas GD. Mean platelet volume: a link between thrombosis and inflammation? Curr Pharm Des. 2011:17(1):47-58.

34. Vega LE, Espinoza LR. Predictors of poor outcome in ANCA-associated Vasculitis (AAV). Curr Rheumatol Rep. 2016;18(12):70.

\section{Publisher's Note}

Springer Nature remains neutral with regard to jurisdictional claims in published maps and institutional affiliations.

Ready to submit your research? Choose BMC and benefit from:

- fast, convenient online submission

- thorough peer review by experienced researchers in your field

- rapid publication on acceptance

- support for research data, including large and complex data types

- gold Open Access which fosters wider collaboration and increased citations

- maximum visibility for your research: over $100 \mathrm{M}$ website views per year

At $\mathrm{BMC}$, research is always in progress.

Learn more biomedcentral.com/submissions 